malarial fever a special class of fevers which are not attributable either to the plasmodium malarie or to Eberth's bacillus, but belong to the auto-infections of the organism. He therefore divides the fevers of Greece into the three following principal classes arranged in order of frequency: (1) Fièvres paludéennes; (2) Fièrres par auto-infection; and (3) Fièvres typhoïdes. La Grrèce Médicale and 'Iarpù̀ II póodos-i.e., Medical Progress, a Greek journal of 36 pagesare published conjointly in Syra, the editor being Dr. Joannes Foustanos.

Friend of China.--In the July number the Rev. J. C. Kerr, M.D., of Canton, has a short paper on the effects of the opium habit, as observed by him in his experience of 40 years "in a large city and in a large hospital."

Mercy and Truth.-In the August number Dr. Mary Synge of Fuh-ning, South China, writes on Women Patients, Rich and Poor, and Mr. Walter Squibbs describes an attack on a medical mission at Kiang-peh in Western China in 1898.

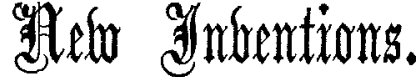

A POCKET CAMEL HAIR SURGICAL BRUSH.

MANY practitioners, in common with myself, must have often found the unsuitableness of the ordinary camel hair brush (for painting throats, \&c.) for being carried in the pocket when on one's rounds. I have designed one on the principle of the pocket penholder, the use of which will be

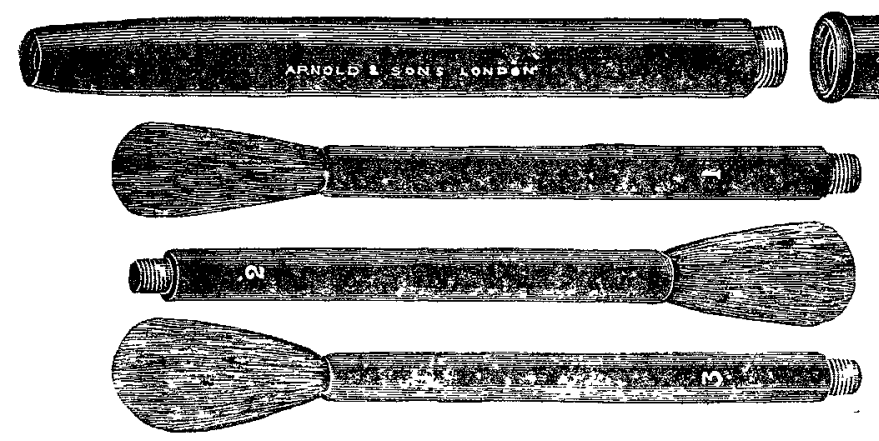

at once apparent. There are two extra brush-ends adaptablo to the vulcanite holder and each one is numerically marked, so that when used for infectious cases, \&c., they can be easily differentiated and " put in quarantine" till thoroughly disinfected. Messrs. Arnold and Sons, surgical instrument makers, of West Smithfield, have carried out my directions secundum artem and a most useful and handy little instrument is the result.

W. L'Heureux Blenkarne, M.R.C.S. ENG., L.S.A. Formerly Surgeon to the Leicester and
Worthing.

\section{THE METROPOLITAN HOSPITAL SUNDAY FUND.}

THE council of the Metropolitan Hospital Sunday Fund met on July 30th at the Mansion House to receive the report of the committee of distribution and to order payment of awards. Sir Sydney H. WATERLow, the Vice-President, occupied the chair.

The following are the awards recommended by the Committee of Distribution for the year 1900 :-

30 General Hospitals.

Charing Cross Hospital, $£ 11693$ s. 4d. ; French Hospital, $£ 325$ 16s. 8d. German Hospital, $\$ 594$ 3s. 4d.: Great Northern Central Hospital, $£ 622$ 18s. 4d.; Guy's Hospital, £1533 68. 8d.; Hampstead Hospital,
$£ 210$ 16s. 8d.; Italian Hospital, £19 3s. 4d. ; King's College Hospital, $£ 210168.8 d$.; Italian Hospital, $£ 19$ 38. 4d. ; King's College Hospital,
$£ 153368.8 d$; London Hospital, £4600; London Homocopathic Hospital, $£ 622$ 18s. 4d.; Phillips Memorial Homoeopathic Hospital, $£ 33$ 10s. 10d.; London Temperance Hospital, 2785 168. 8d. : Metropolitan Hospital,
f690; Mildmay Hospital, £258 15s.; Miller Hospital and Royal Kent £690; Mildmay Hospital, £258 158.; Miller Hospital and Royal Kent
Dispensary, £268 6s.8d.; North-West Lonton Hospital, £383 6s. 8d. ;
Poplar Hospital, £622 18s. 4cl. ; Roval Free Hospital, £1006 5s.; St. George's Hospital, £1935 168. 8d.; SS. John and Elizabeth Hospital $£ 47$ 18s. 4d. ; St. Marv's Hospital, £1974 3s. 4d. ; St. Thomas's Hospital, $£ 43158$. ; Seamen's Hospital Society, £1111 13s. 4d.; the Middlesex Hospital, £2012 10*.; Tottenham Hospital, £191 13.; 4d.; University College Hocpital, $£ 1245$ 16.8 8d ; Walthamstow. $£ 86$ 58.; West Ham Hospital, $£ 392$ 18s. $4 d$; West London Hospital, $£ 622$ 18s. $4 d$; ; and Westminster Hospital, £1341 13s. 4 d.

Special Hospitals : 5 Chest Hospitals.

City of London Hospital for Diseases of the Chest, Victoria-park $£ 670$ 16s. 8d.; Hospital for Consumption, Brompton, $£ 1820$ l6s. 8d. North London Consumption Hospital, Hampstead, $£^{3} 643$ s. 4d. ; Roya Hospital for Diseases of the Chest, City-road, $£ 3836 s .8 d$; and Royal National Hospital for Consumption, Ventnor, $£ 28710$.

17 CHILDREN's Hospitals.

Alexandra Hospital for Hip Disease, W.C., \&143 15s.; Banstead Surgical Home, $£ 38$ 6. 8d. ; Barnet Home Hospital, £38 6s. 8d.; Belgrave Hospital for Children, S.W., £124 118. 8d.; Cheyne Hospita for Incurable Children, S.W., $£ 9516 s$. $8 d$.; Hast London Hospital for Children, Shadwell, $\mathbf{E}, £ 651$ 13\%. 4d.; Evelina Hospital for Sick Children, Southwark, S. E.. $£ 3836 s$. $8 d$.; Home for Incurable Children, Maida-vale, W.. \&76 13\&. A $i$. ; Home for Sick Children, Sydenham, S.E. £124 11s. 8d.; Hospital for Sick Children, Great Ormond-street, W.C. $£ 910$ 8s. 4d. ; North-Eastern Hospital for Children, Hackney-road,

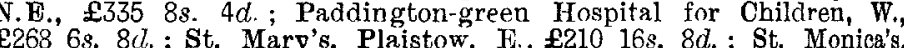
Brondesbury, N.W., $£ 100$ 12s. $6 d$. ; Victoria Hospital for Children, King's-road, Chelsea, s.W., $\$ 575$; Victoria Home, Margate, £23 19s. 2d.; and "The Vine," Sevenoaks, £38 6s.8d.

6 Lxing-in Hospitals.

British Lying-in Hospital, Endell-street, W.C., £33 10s. 10d.; City of London Lying-in Hospital, City-roar, E.C., 247 18s. 4d.; Clapham Maternity Hospital, £28 15s.; East Fnd Mothers Home, £81 9s.2d. General Lying-in Hospital, Lambeth, S.E., £38 6s. 8d. ; Queen Charlotte's lying-in Hospital, Marylebone-road, W., £335 $88.4 d$. 6 HOSPITALS FOR WOMEN.

Chelsea Hospital for Women, S.W., £364 3s. 4d. ; Hospital for Women Soho-square. W., £239 11s. 8d.; Grosvenor Hospital for Women and Children, Vincent-square, S. W., $£ 1058 s$. 4d. ; New Hospital for Children and Women, Lambeth, S.E., £215 12s. 6d.; Samaritan Free Hospital, Lower Seymour-street, W., £383 6s. 8d.

27 Other Special Hospitats.

Cancer Hospital, Brompton, S.W., 2575 ; London Fever Hospital, Islington, N., $£ 670$ 16s. 8d. ; Gordon Hospital for Fistula, Vauxhall Bridge-road, S.W., f28 158.; St. Mark's Hospital for Fistula, City-road, E.C., nil; National Hospital for the Diseases of the Heart, Soho square, $W ., £ 76$ 138. $4 d$.; Female Lock Hospital, Harrow-road, W., square, W., $£ 76$ 13s. 4d.; Female Lock Hospital, Harrow-road, W,
\&239 11s. $8 d$.; Hospital for Epilepsy, Paralysis, and Other Diseases of f239 11s. 8d. ; Hospital for Epilepsy, Paralysis, and Other Diseases of
the Nervous System, Regent's Park, N.W., £52 148. 2d.; National Hospital for the Paralysed and Epileptic, W.C., deferred; West Hospital for the Paralysed and Epileptic, W.C., deferred; West End Hospital for Diseases of the Nervous Sustem, £115; Central London Ophthalmic Hospital, Gray's Inn-road, W.C., $£ 5710 s$,
Royal Eye Hospital, St. George's-circus, S.F., $£ 17210$ s. ; Royal London Troyal Eye Hospital, St. George's-circus, S. F., $£ 17210$ s. ; Royal London
Ophthalmic Hospital, City-road, E.C., $£ 575 ;$ Royal Westminster Ophthalmic Hospital, City-road, E.C., £575; Royal Westminster
Ophthalmic Hospital, Charing Cross, W.C., £115; Western Ophthalmic Ophthalmic Hospital, Charing Cross, W.C., £115; Western Ophthalmic
Hospital, Marylebone-road, W., $£ 3868.8 d$.; City Orthopædic Hospital, Hospital, Marylebone-road, W., £38 68. 8d. ; City Orthopædic Hospital, Hatton-garden, E.C. £115; National Orthopædic Hospital, Great Portland-street, W., \&105 8s. 4d.; Royal Orthopædic Hospital, Oxford-
street, W., £95 16s. 8d. ; Rnyal Sea-Bathing Hospital, Margate, £287 10s.; street, W., £95 16s.8d.; Royal Sea-Bathing Hospital, Margate, £28710s.
St. John's Hospital for Diseases of the Skin, £76 138. 4d.; Hospital for Diseases of the Skin, Stamford-street, S.E., nil ; St. Peter's Hospital for Stone, Covent-garden, $£ 7613 \mathrm{~s}$. $4 d$.; Central London Throat and Ear Hospital, Gray's Inn-road, W.C., $£ 33$ 10s. 10d.; London Throat Hospital Great Portland-street, W., $£ 3310 s .10 d$.; Metropolitan War, Nose, and Throat Hospital, Grafton-street, W., £911s. 8d. ; Royal Ear Hospital Frith-street, W., $£ 9$ 11s.8d.; Dental Hospital of London, Teicestersquare, W.C. $£ 115 ;$ National Dental Hospital, 149, Great Portland-
street, W., $\$ 47188$. 4 .

29 Convalescent Hospitals. Metropolitan Convalescent Institution, Sackville-street, W., 2603158 ;
Bexhill Convalescent Home, Sackville-street, W., £335 8s. 4d.; All Saints' Convalescent Hospita], Eastbourne, £383 6s. 8d.; Ascot Priory Convalescent Home, $£ 67$ 1s. 8d. ; Charing Oross Hospital Convalescent Home, Limpsfield, $£ 67$ 1s. 8d.; Chelsea Hospital for Women Convalescent Home, St. Leonards, $£ 432$ 2s. 6 d. ; Hahnemann Convalescent Home, Bournemouth, £23 19s. 2d.; Hanwell Convalescent Home, £26 16s. 8d.; Herbert Convalescent Home, Bournemouth, £23 198. 2d. ; Herne Bay Baldwin-Brown Convalescent Home, $£ 33108$. 10d. ; Herne Bay Mothers' Home, $1405 s$; ; Homceopathic Convalescent Home,
Fastbourne, $£ 193 \mathrm{~s}$. 4d.; King's College Hospital Convalescent Home, Hemel Hempstead, £57 10s.; Mrs. Kitto's Convalescent Home, Reigate, Hemel Hempstead, £57 10s.; Mrs. Kitto's Convalescent Home, Reigate, $£ 67$ 1s. 8d. ; London and Brighton Female Convalescent Home,
$£ 9$ 11s. 8d. ; Mary Wardell Convalescent Home for Scarlet Fever, $£ 9$ 11s. 8d.; Mary Wardell Convalescent Home for Scarlet Fever, $£ 105$ 8s. 4d.; Morley House Convalescent Home for Working Men,
$£ 177$ 5s. 10 .; Police Seaside Home, Brighton, £47 18s. 4d.; St. $£ 177$ 5s. 10d.; Police Seaside Home, Brighton, £47 18s. 4d.; St. Andrew's Convalescent Hospital, Clewer, $£ 143$ 15s. : St. Andrew's Con-
valescent Home, Folkestone, $£ 2208 s .4 d$. ; St. John's Home for Convavalescent Home, Folkestone, £220 8s. 4d. ; St. John's Home for Convalescent and Crippled Children, Brighton, $£ 95$ 16s. 8d.; St. Joseph's Con-
valescent Home, Bournemouth, $£ 47188.4 d$; St. Leonards-on-Sea Convalescent Home, Bournemouth, $£ 47188.4 d$; St. Leonards-on-Sea Convalescent Home for Poor Children, £95 $168.8 d$.; St. Mary's Convalescent
Home, Shortlands, $£ 17$ 5s.; St. Michael's Convalescent Home, Home, Shortlands, £17 5s. ; St. Michael's Convalescent Home, Westgate-on-Sea, $£ 3310$ s. 10d. ; Seaside Convalescent Hospital, Seaford, $£ 9516 s .8 d$. ; Warley Convalescent Cottage Hospital, Essex, £10 10s. 10d.;
Friendly Societies' Convalescent Home, Dover, £19 3s. 4d.; Deptford Medical Mission Convalescent Home, Bexhill, £19 3s. $4 d$.

\section{CotTage Hospitals.}

Beckenham Cottage Hospital, $£ 47188.4 d$.; Blackheath and Charlton Cottage Hospital, 117 17s. 6d. ; Bromley, Kent, Cottage Hospital, 283 3. 4d.; Chislehurst, Sidcup and Cray Valley Cottag 9 Hospital, £38 6s. 8d.; Tltham Cottage Hospital, £30 13s. 4d.; Enfteld Cottage Hospital, 252 14s. 2d. ; Epsom and Ewell Cottage Hospital, £57 10s.; Hounslow Cottage Hospital, $22208.10 d$. ; Livingstone Cottage Hospital
$£ 57$ 10s. ; Mildmay Cottage Hospital, $£ 57$ 10s. ; Reigate and Redhill 